APPLICAZIONE DI UN PROTOCOLLO ONE-STEP REAL TIME RT-PCR PER LA RICERCA DI NOROVIRUS IN MOLLUSCHI BIVALVE RACCOLTI E COMMERCIALIZZATI NELLA REGIONE CAMPANIA

\title{
APPLICATION OF A ONE-STEP REAL TIME RT-PCR PROTOCOL FOR NOROVIRUS DETECTION IN SHELLFISH HARVERSTED AND COMMERCIALIZED IN CAMPANIA REGION
}

\author{
Pepe T. ${ }^{1}$, Ventrone I. ${ }^{1}$, Suffredini E. ${ }^{2}$, Ceruso M. ${ }^{1}$, Croci L. ${ }^{2}$, Anastasio A. ${ }^{1}$ \\ (1) Università degli Studi di Napoli "Federico II", Dipartimento di Scienze Zootecniche e Ispezione degli Alimenti, Italy \\ (2) Istituto Superiore di Sanità, Dipartimento di Sanità Pubblica Veterinaria e Sicurezza Alimentare, Italy
}

SUMMARY

Key words

\begin{abstract}
Shellfish are recognized as a potential vehicle of viral diseases. However, according to European Regulations, only bacteriological parameters (Salmonella and E. coli) are used to establish their microbiological quality. Because of the lack of a standard reference method for the detection of viruses, data on the incidence of enteric viruses in European production areas are still limited. Aim of the present study was the evaluation of the Norovirus (NoV) contamination in shellfish from different harvesting areas (both class $\mathrm{A}$ and $\mathrm{B}$ ) and from registered and not- registered retails of the Campania region (Tyrrhenian Sea, Italy). Seventy shellfish (Mytilus galloprovincialis) were collected from 15 harvesting areas, one authorized and two not authorized retails and were examined for virus presence (NoV genogroups I and II) by Real Time PCR. Ten harvesting areas (66\%) showed presence of viral contamination, with three of them belonging to A areas and seven to B areas. The shellfish collected from the registered retail tested positive for Norovirus presence, whereas the shellfish collected from not-authorized retails didn't show viral contamination. Twenty-nine samples were positive for genogroup II, ten of which tested positive also for NoV genogroup I.
\end{abstract}

Shellfish, Norovirus, Real-Time.

\section{INTRODUZIONE}

Nel corso del 2006 sono pervenute al sistema d'allerta comunitario per alimenti e mangimi nove notifiche riguardanti episodi di gastroenterite verificatisi in consumatori che avevano ingerito alimenti contaminati da Norovirus (1), ritenuti una delle cause principali di gastroenterite infettiva umana $(2,3)$.

Le informazioni epidemiologiche sulle gastroenteriti virali nel nostro Paese sono scarse e frammentarie e ciò potrebbe portare a sottostimare il numero effettivo di casi che si verificano annualmente.

Sulla base della distanza filogenetica i Norovirus sono stati suddivisi in 5 differenti genogruppi (GI, GII, GIII, GIV, GV) ciascuno dei quali comprende di- versi genotipi. I genogruppi GI, GII e GIV sono responsabili di infezioni nell'uomo. Il GII è da collegare alla maggior parte dei casi di gastroenterite da Norovirus registrati $(92 \%)$, i restanti casi sono attribuiti al GI e, in subordine, al GIV (3).

Considerata la rilevanza assunta da tale malattia in questi ultimi anni, la Direzione Generale per la Sicurezza degli Alimenti e della Nutrizione del Ministero della Salute ha ritenuto opportuno mettere a disposizione delle autorità sanitarie di controllo un documento guida, elaborato appositamente dall'Istituto Superiore di Sanità, nel quale vengono anche forniti orientamenti per la ricerca dei Norovirus nei campioni di feci e di alimenti (1). Nel documento in questione vengono elencati i criteri da seguire per effettuare questo tipo di indagini virolo- 
giche. La Real Time RT-PCR è stata indicata fra le metodiche di analisi prese in considerazione per la determinazione dei Norovirus (1).

Scopo della presente indagine, svolta in collaborazione con l'ISS, è stato valutare la presenza di Norovirus in un comune veicolo di diffusione delle gastroenteriti da Norovirus (4), i molluschi bivalvi, prodotti nella Regione Campania. L'analisi è stata condotta mediante applicazione di un protocollo onestep Real Time RT-PCR.

\section{MATERIALI E METODI}

Sono stati prelevati n. 67 campioni di molluschi bivalvi (Mytilus galloprovincialis) da n. 15 allevamenti posti lungo le coste della regione Campania. Sono stati inoltre prelevati $\mathrm{n}$. 1 campione da un punto vendita autorizzato e n. 2 campioni da venditori abusivi. Per quanto riguarda gli allevamenti, 7 allevamenti erano situati in aree di classe A e 8 allevamenti in aree di classe B. La posizione dei siti di campionamento è riportata in Figura 1.

Da 9 allevamenti oggetto di controllo (6 di area A e 3 di area B) sono state prelevate 5 aliquote, 4 nei punti cardinali delimitanti l'allevamento e 1 al centro dello stesso. Da 5 allevamenti ( 1 di area A e 4 di area B) le aliquote sono state prelevate dai 4 punti cardinali e da un allevamento di area $\mathrm{B}$ il prelievo è stato effettuato in soli 2 punti (nord-est e sud-est). Ciascuna aliquota era composta da un pool di mitili provenienti dalle porzioni alta, media e bassa di ogni filare. I campionamenti sono stati effettuati in condizioni ideali, potendo in tal modo escludere che risultati difformi potessero essere determinati da situazioni climatiche non favorevoli.
I campioni sono stati trasportati mediante contenitore isotermico refrigerato presso i laboratori della Sezione di Ispezione degli Alimenti di Origine Animale e immediatamente analizzati. Per ogni pool campionato sono stati selezionati n. 10 esemplari dai quali è stato prelevato, e finemente sminuzzato mediante bisturi sterile, l'epatopancreas. Di questo 2.0 g sono stati addizionati di $2.0 \mathrm{ml}$ di soluzione di proteinasi $\mathrm{K}(0.1 \mathrm{mg} / \mathrm{ml})$, incubati a $37^{\circ} \mathrm{C}$ con agitazione per 60 min e successivamente sottoposti ad una seconda incubazione a $65^{\circ} \mathrm{C}$ per $15 \mathrm{~min}$ in bagnomaria. L'estrazione degli acidi nucleici è stata effettuata mediante NucliSense MiniMag extraction kit (bioMeriuex) (5). La combinazione dei primers e probes è stata scelta dalla letteratura (6). La retrotrascrizione e la PCR one-step sono state effettuate con ABI Prism 7700 SDS detector (Applied Biosystems) mediante l'utilizzo del kit Platinum ${ }^{\circledR}$ Quantitative RTPCR ThermoScriptTM One-Step System (Invitrogen). Per ogni reazione $5 \mathrm{l}$ di acido nucleico sono stati aggiunti ad un mix di 201 contenente $1 \mathrm{X}$ di buffer, $250 \mathrm{nmol} / \mathrm{L}$ del probe, $900 \mathrm{nmol} / \mathrm{L}$ del primer reverse e $500 \mathrm{nmol} / \mathrm{L}$ del forward, $1 \mathrm{X}$ ROX (reference dye), $2.5 \mathrm{U}$ di Thermoscript Plus/Platinum Taq e 2 U di inibitore delle RNAsi (Eppendorf). ROX è stato utilizzato per la normalizzazione del segnale. La retrotrascrizione è stata effettuata a $55^{\circ} \mathrm{C}$ per $60 \mathrm{~min}$, seguita da denaturazione a $95^{\circ} \mathrm{C}$ per 5 min e da 45 cicli di PCR (denaturazione a $95^{\circ} \mathrm{C}$ per $15 \mathrm{~s}$, annealing a $60^{\circ} \mathrm{C}$ per $1 \mathrm{~min}$, extension a $65^{\circ} \mathrm{C}$ per $1 \mathrm{~min}$ ). Ad ogni corsa sono stati affiancati due controlli negativi e un controllo positivo costituito da RNA estratto da Norovirus. Tutti i campioni sono stati testati in tre repliche in saggi indipendenti. Il campione è stato considerato positivo quando un $\mathrm{Ct}$ inferiore a 44 era presente in almeno due repliche (Fig. 2).
Figura 1: Punti di prelievo.

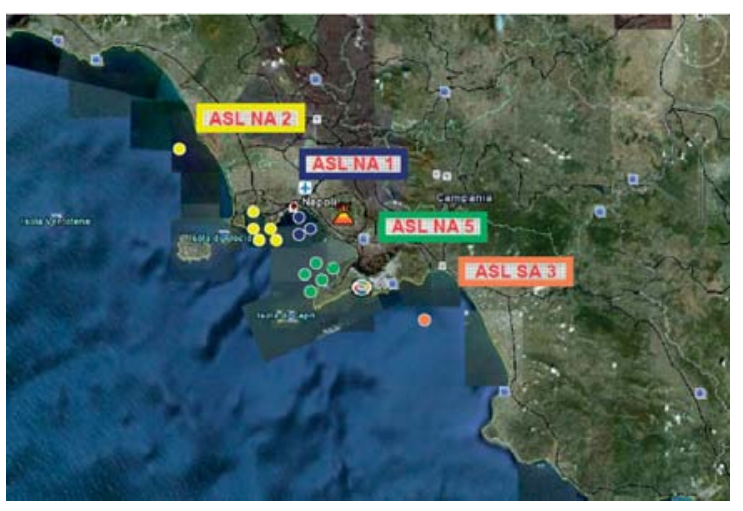

Figura 2: Amplificazioni in Real-Time di Norovirus

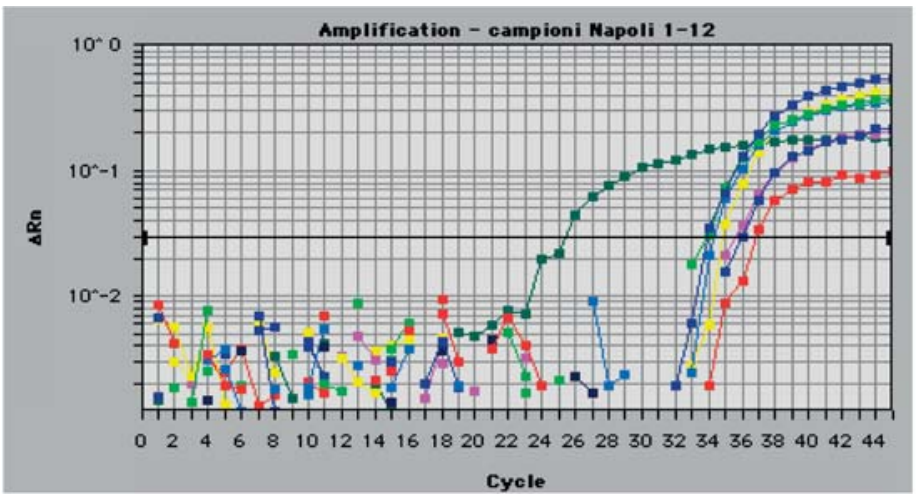




\section{RISULTATI}

La tecnica One-step Real Time RT-PCR ha consentito di evidenziare la presenza di Norovirus nei campioni esaminati.

Dieci dei quindici allevamenti campionati $(66 \%)$ sono risultati positivi per la presenza di Norovirus. In particolare, solo uno degli otto allevamenti presenti in aree di classe $B$ è risultato privo di contaminazione in tutti i punti di prelievo, cinque allevamenti hanno evidenziato positività per i Norovirus in tutti i punti di campionamento e due hanno rilevato la presenza del virus solo per alcuni punti campionati. Per quanto riguarda i sette allevamenti siti in aree di classe A, uno di essi ha evidenziato positività in tutti i punti di prelievo mentre in due allevamenti è stata riscontrata la presenza di Norovirus soltanto in uno dei cinque punti di prelievo (Tab. 1).

Il campione di mitili proveniente dal punto vendita autorizzato è risultato positivo, diversamente il virus non è stato evidenziato nei campioni provenienti dai punti vendita non autorizzati. In totale $\mathrm{i}$ campioni positivi sono stati n. $29(41 \%)$, di cui n. 6 campioni provenienti da allevamenti di area A, n. 22 da allevamenti di area $\mathrm{B}$ e $\mathrm{n} .1$ da un punto vendita autorizzato. La totalità dei campioni è risultata po- sitiva per il GII, di questi il 34\% (n. 10 campioni su n. 29) era positivo anche per il GI. Cinque campioni positivi per entrambi i genogruppi provenivano da allevamenti di area A, quattro da allevamenti di area B ed un campione proveniva dal solo punto di vendita autorizzato campionato.

\section{CONSIDERAZIONI E CONCLUSIONI}

Per la ricerca dei Norovirus la metodica molecolare è stata considerata la più affidabile dal Ministero della Salute che ne ha raccomandato l'applicazione in una nota del 20/04/2007 (1). I risultati ottenuti hanno confermato che la tecnica One-step Real Time RT-PCR è efficace per la loro ricerca. I dati evidenziano una vasta circolazione di Norovirus in aree dedicate alla produzione di molluschi bivalvi.

La presenza di particelle virali in molluschi provenienti da zone classificate A conferma che i batteri fecali non possono essere considerati indici affidabili per la presenza di Norovirus in questi alimenti (7).

Il rinvenimento di Norovirus in campioni provenienti da un punto vendita autorizzato conferma la

Tabella 1: Allevamenti campionati

\begin{tabular}{|c|c|c|c|c|c|}
\hline Allevamenti & \multicolumn{5}{|c|}{ Prelievi: pool delle tre profondità di ciascun filare } \\
\hline Zona A & N-E & N-O & Centro & S-E & S-O \\
\hline 1 & + & + & & + & + \\
\hline 2 & - & - & - & - & + \\
\hline 3 & - & - & - & - & - \\
\hline 4 & - & - & - & - & - \\
\hline 5 & - & - & - & - & - \\
\hline 6 & - & - & - & + & + \\
\hline 7 & - & - & - & - & + \\
\hline Zona B & + & + & + & + & - \\
\hline 1 & + & - & - & + & + \\
\hline 2 & - & - & & + & + \\
\hline 3 & + & + & + & + & + \\
\hline 4 & + & + & & + & + \\
\hline 5 & + & + & & + & + \\
\hline 6 & + & + & & + & + \\
\hline 7 & + & & & & + \\
\hline
\end{tabular}


necessità di effettuare controlli lungo tutta la filiera produttiva dei molluschi bivalve.

È necessario altresì mettere a punto metodi di analisi standardizzati per la ricerca dei virus enterici, al fine di ottenere risultati univoci e tra loro comparabili.

\section{BIBLIOGRAFIA}

1) Circolare del Ministero della Salute. Direzione generale per la sicurezza degli alimenti e della nutrizione del 20 aprile 2007. Prot. DGSAN/ VIII (EX VI) 3734.

2) Noel, J.S; Fankauser, R.L; Ando, T; Monroe, S.S; Glass, R.I. (1999). Identification of a distinct common strain of "Norwalk-like viruses" having a global distribution. The Journal of Infectious Diseases, 179, 1334-1344.

3) Boccia, D; Tozzi, A.E; Cotter, B; Rizzo, C; Russo, T; Buttinelli, G; Caprioli, A; Marziano, M.L; Ruggeri F.M. (2002) Waterborne outbreak of Norwalk-like virus gastroenteritis at a tourist resort, Italy. Emerging Infectious Diseases, 8(6), 563-568.

4) Nakagawa-Okamoto, R. ; Arita-Nishida, T; Toda, S; Kato, H; Iwata, H; Akiyama, M; Nishio, O; Kimura, H; Noda, M; Takeda, N; Oka, T. (2009). Detection of multiple Sapovirus genotypes and genogroups in oyster-associated outbreaks. Japanese Journal of Infectious Diseases, 62, 6366.

5) Boom, R; Sol, C.J.A; Salimans, M.M.M; Jansen, C.L; Wertheim-van Dillen, P.M.E; Van Der Noordaa, J. (1989) . Rapid and simple method for purification of nucleic acids. Journal of Clinical Microbiology, 28, 495-503.

6) da Silva, A.K; Le Saux, J.C; Parnaudeau, S; Pommepuy, M; Elimelech, M; Le Guyader, F.S. (2007). Evaluation of removal of Noroviruses during wastewater treatment, using Real-Time Reverse Transcription-PCR : different behaviors of Genogroups I and II. Applied Environmental Microbiology, 73, 7891-7897.

7) Commission Regulation (EC) No 2073/2005 of 15 November 2005 on microbiological criteria for foodstuffs. Official Journal of the European Union, L338, 6-7. 